\title{
Análise bifator da Escala Baptista de Depressão - Versão Idosos (EBADEP-ID)
}

\author{
Makilim Nunes Baptista ${ }^{1}{ }^{1}$ \\ Luísa Milani Santos ${ }^{2}{ }^{2}$ \\ Nelson Hauck Filho ${ }^{1}{ }^{1}$ \\ ${ }^{1}$ Universidade São Francisco. Campinas, SP, Brasil. \\ ${ }^{2}$ Universidade Estadual de Campinas. Campinas, SP, Brasil.
}

\begin{abstract}
Resumo
Modelos bifator têm sido relatados na literatura como explicações plausíveis para a estrutura latente da depressão. O presente estudo busca expandir essa área de investigação, tendo como objetivo principal realizar uma análise exploratória bifator da Escala Baptista de Depressão - Versão Idosos (EBADEP-ID). Os participantes foram 311 idosos com idades variando de 60 a 90 anos, provenientes de cinco grupos populacionais de tipo clínico e não clínico. Os resultados mostraram um melhor ajuste aos dados para o modelo bifator com um fator geral e dois fatores específicos, quando comparado a uma simples solução unidimensional. Entretanto, todos os itens carregaram predominantemente no fator geral, ocorrendo poucas cargas significativas nos fatores específicos, o que sustenta a unidimensionalidade do instrumento. O escore geral da EBADEP-ID apresentou elevada consistência interna ( 0,95 pelo coeficiente alfa, e 0,98 pelo coeficiente ômega) e capacidade informativa, além de alta sensibilidade e especificidade. Implicações teóricas e práticas dos resultados, bem como limitações do estudo, são discutidas ao final.
\end{abstract}

Palavras-chave: transtorno de humor, testes psicológicos, dimensionalidade.

\section{Bifactor analysis of the Baptista's Depression Scale}

\begin{abstract}
Bifactor models have been reported in the literature as plausible explanations of the latente structure of depression. In the present study, we offer a contribution to this area of research, by performing an exploratory bifactor analysis of the Baptista's Depression Scale-elderly version (EBADEP-ID). Participants were 311 elderlies with ages ranging from 60 to 90 years, coming from five distinct clinical and nonclinical populations. The bifactor model comprising one general factor and two specific factors yielded a better fit to the data when compared with a simple unidimensional model. However, all items loaded highly on the general factor, and few items had loadings on the specific factors in the model, then supporting the unidimensionality of the EBADEP-ID. The sum score provided by the instrument achieved high internal consistency (.95 according to alpha, and .98 according to ômega coefficient) and informative capacity, besides high sensitivity and specificity. Theoretical and practical implications of the findings, as well as limitations to the study, are discussed.
\end{abstract}

Keywords: affective disorders, psychological tests, dimensionality.

\section{Análisis bifactor de la Escala Baptista de Depresión}

\section{Resumen}

Los modelos bifator han sido reportados en la literatura como explicaciones plausibles para la estructura latente de la depresión. El presente estudio busca expandir esa área de investigación, teniendo como objetivo principal realizar un análisis exploratorio bifator de la Escala Baptista de Depressão-Versão Idosos (EBADEP-ID). Los participantes fueron 311 ancianos con edades variando de 60 a 90 años, provenientes de cinco grupos poblacionales de tipo clínico y no clínico. Los resultados mostraron un mejor ajuste a los datos para el modelo bifator con un factor general y dos factores específicos, en comparación con una simple solución unidimensional. Sin embargo, todos los elementos cargaron predominantemente en el factor general, ocurriendo pocas cargas significativas en los factores específicos, lo que sostiene la unidimensionalidad del instrumento. La puntuación general de EBADEP-ID presentó elevada consistencia interna (0,95 por el coeficiente alfa, y 0,98 por el coeficiente omega) y capacidad informativa, además de alta sensibilidad y especificidad. Las implicaciones teóricas y prácticas de los resultados, así como las limitaciones del estudio, se discuten al final.

Palabras clave: trastorno de humor, pruebas psicológicas, dimensionalidad. 
O aumento na disponibilidade de recursos tem levado a um envelhecimento da população mundial. Em 2002, um levantamento da World Health Organization (2002) estimou a existência de aproximadamente 600 milhões de idosos em todo o mundo, além de uma expectativa de um bilhão para 2020. Segundo o Instituto Brasileiro de Geografia e Estatística (2002), no Brasil, em 2002, idosos representavam 8,6\% da população total (aproximadamente 15 milhões), sendo previstos $13 \%$ da população total em 2020 (30 milhões). Diante desse cenário, torna-se importante a identificação de problemas de saúde geral/mental, orientação e acompanhamento dos problemas mais comuns na terceira idade (Almeida et al., 1997), sendo alguns dos principais problemas os déficits cognitivos e os transtornos de humor (Bottino, Blay, \& Laks, 2012; Silberman et al., 1995). O presente estudo busca trazer uma contribuição à área, abordando um aspecto específico da depressão, a estrutura fatorial de um instrumento voltado à população de idosos.

A depressão é uma doença com prevalência relativamente alta na terceira idade em comparação às demais faixas etárias. Estimativas são de que a depressão chega a acometer $20 \%$ dos idosos em países ocidentais (Volkert, Schulz, Härter, Wlodarczyk, \& Andreas, 2013), sendo especialmente frequentes em indivíduos com declínio cognitivo (Polyakova et al., 2014), pessoas internadas em instituição de longa permanência (Achterberg, Pot, Kerkstra, \& Ribbe, 2006) e pacientes hospitalizados (Walker et al., 2016). A depressão, bem como o risco de suicídio, é mais alta no final do ciclo vital quando comparada a outras etapas da vida, e pode ocorrer em função de fatores tais como declínio cognitivo, dificuldades com a tecnologia, problemas financeiros, preconceito contra a terceira idade, mudança nas estruturas de papéis parentais e profissionais, entre outros. Nessa população, as principais características da depressão parecem ser a disforia, o isolamento social, a deterioração cognitiva, a apatia e o humor positivo reduzido (Kim, DeCoster, Huang, \& Bryant, 2013).

Existem diversos instrumentos psicométricos desenvolvidos para avaliar sintomas de depressão em idosos e indivíduos de outras faixas etárias (Baptista \& Borges, 2016). Apenas para mencionar, em 2006, já estavam disponíveis 280 medidas diferentes publicadas de 1940 a 2000, com considerável variabilidade na cobertura de faixas etárias (Santor, Gregus, \& Welch, 2006). Baptista e Borges (2016) localizaram 24 instrumentos que foram utilizados para avaliar sintomas depressivos em estudos nacionais. Dentre eles, alguns dos mais populares para uso na população de idosos são o Beck Depression Inventory-
II (ver Argimon, Paloski, Farina, \& Irigaray, 2016) e o Geriatric Depression Scale (Pocklington, Gilbody, Manea, \& McMillan, 2016). Essas ferramentas podem variar no quanto enfatizam cada uma das dimensões clássicas da depressão, que são a afetiva/humor (ex., Montgomery-Asberg Depression Rating Scale, Montgomery \& Åsberg, 1979), a cognitiva (Beck Depression Inventory, Beck, Ward, Mendelson, Mock, \& Erbaugh, 1961) e a somática/vegetativa (Hamilton Rating Scale for Depression, Hamilton, 1967) (para uma revisão, ver Baptista, 2012).

Diferentes modelos teóricos e estatísticos foram propostos para auxiliar na compreensão da estrutura latente da depressão, independentemente da faixa etária (Schmittmann et al., 2013). Não obstante, evidências recentes têm sugerido a existência de um fator geral de depressão. Ao efetuar uma meta-análise da estrutura fatorial de quatro das escalas mais utilizadas internacionalmente Shafer (2006) encontrou, em todos os instrumentos, um fator geral predominante, ainda que fatores específicos (aspectos afetivos, cognitivos ou somáticos) também tenham emergido. Esse fator geral representa uma vulnerabilidade à depressão e quiçá também a outros transtornos mentais (Baptista, Hauck Filho, \& Cardoso, 2016; Borges, Baptista, \& Serpa, 2017). A ideia de um fator geral implica em um modelo reflexivo de variáveis latentes, ou seja, esse fator é entendido e modelado como a causa latente para os diversos sintomas da depressão (Schmittmann et al., 2013).

Uma abordagem que concilia a existência de um fator geral e de outros fatores mais específicos é o modelo bifator. Um modelo bifator é aquele em que um conjunto de descritores é explicado por um fator geral e, simultaneamente, por um ou mais fatores específicos, ortogonais ao fator geral (Reise, Moore, \& Haviland, 2010). Ele é utilizado para testar a hipótese da existência de uma vulnerabilidade geral ao transtorno em questão, podendo também revelar outros componentes de risco independentes, mas de menor impacto. Embora modelos bifator remontem ao início da Psicometria (Spearman, 1904), apenas recentemente começaram a ter maior aplicação no contexto da saúde mental (Reise, Morizot, \& Hays, 2007).

Ao serem aplicados no contexto da depressão, modelos bifator tendem a apresentar o melhor ajuste aos dados quando comparados com outras possibilidades de dimensionalidade. Por exemplo, no caso do BDIII, foi relatado um melhor ajuste e coerência teórica para um modelo bifator composto por um fator geral e três fatores específicos (afetivo, cognitivo e somático), tanto para adolescentes (Osman, Barrios, Gutierrez, Williams, \& Bailey, 2008) quanto para adultos 
(Brouwer, Meijer, \& Zevalkink, 2013). Um modelo bifator também foi encontrado para a Hospital Anxiety and Depression Scale em amostras com participantes adultos de grande variabilidade etária (Iani, Lauriola, \& Costantini, 2014; Norton, Cosco, Doyle, Done, \& Sacker, 2013), para a Depression Anxiety Stress Scales-21 em universitários (Osman et al., 2012) e para a Center for Epidemiologic Studies Depression Scale em idosos (Gomez \& McLaren, 2015). Vale ressaltar que esse modelo parece representar melhor a estrutura de instrumentos de depressão não apenas em muitos países do ocidente (Burns, Höfer, Curry, Sexton, \& Doyle, 2014), mas também em culturas orientais (Chin et al., 2015).

Um instrumento recentemente publicado destinado à avaliação de sintomas do quadro em idosos é a Escala Baptista de Depressão Versão Idosos (EBADEP-ID; Coutinho, Hamdan, \& Baptista, 2016). A EBADEPID teve seus itens desenvolvidos a partir dos critérios do Manual Diagnóstico e Estatístico de Desordens Mentais - DSM-5 (American Psychiatric Association, 2013), da Classificação Internacional de doenças CID (Organização Mundial de Saúde [OMS], 1995), e também com base na teoria cognitiva de Beck (Beck, Rush, Shaw, \& Emery, 1987) e na teoria comportamental da depressão (Ferster, Culbertson, \& Boren, 1997). Na versão preliminar do trabalho de Coutinho, Hamdan e Baptista (2016), a ferramenta ficou composta por 70 itens dicotômicos avaliativos de um fator geral de depressão. Trata-se de uma adaptação da EBADEP versão adultos (EBADEP-A; Baptista, 2012), altamente correlacionada $(r=0,76)$ à Geriatric Depression Scale (uma versão de 32 itens da EBADEP-ID se correlacionou em 0,79 com o mesmo instrumento; Carneiro, Baptista, \& Santos, 2013), e capaz de discriminar idosos com e sem diagnóstico de depressão $(d=1,57)$. Em outro estudo, a EBADEPID apresentou uma correlação negativa moderada $(r=-0,57)$ com uma medida de qualidade de vida (da Silva \& Baptista, 2016). Assim, embora recente, trata-se de uma escala bastante promissora para a avaliação da depressão na população de idosos brasileira.
Uma possibilidade ainda inexplorada diz respeito à plausibilidade de uma estrutura bifator para a EBADEPID. Tanto a EBADEP-ID quanto a EBADEP-A foram elaboradas de modo a contemplar uma variabilidade de aspectos afetivos, cognitivos, somáticos e sociais da depressão. Por isso, é possível que fatores residuais, para além de um fator geral, estejam presentes enquanto variáveis que influenciam nas respostas aos itens, capturando a variância comum entre descritores de um mesmo agrupamento teórico (ex., afetivo). Dependendo da saliência desses fatores específicos, pode ficar comprometido o uso do instrumento com base apenas em um fator geral de depressão, sendo mais recomendável o cômputo de escores separados para cada fator. Em virtude disso, o presente estudo teve como objetivo principal realizar uma análise exploratória bifator da EBADEP-ID, comparando a solução encontrada com um modelo unidimensional. Para além de testar a estrutura de um instrumento particular, o estudo busca aprofundar o entendimento do fenômeno no Brasil, sendo um dos primeiros estudos (senão o primeiro) a endereçar a questão em um instrumento de depressão em idosos. São também exploradas outras propriedades psicométricas da escala, como sua fidedignidade, sensibilidade e especificidade.

\section{Método}

\section{Participantes}

A amostra total de participantes do presente estudo foi de 311 idosos com idades variando de 60 a 90 anos $(M=70,6$ anos, $D P=7,7)$, sendo $57 \%$ mulheres. Essa amostra foi composta por cinco grupos distintos de indivíduos, tais como apresentados na Tabela 1. O primeiro grupo foi composto por 62 idosos $(\mathrm{M}=71,5$; $\mathrm{DP}=7,6$ anos; $80,1 \%$ feminino) de grupos de idosos da prefeitura de uma cidade do interior de São Paulo. O segundo grupo foi composto por 40 pacientes internados em um hospital da cidade de Curitiba, $\mathrm{PR},(\mathrm{M}=72,3$; $\mathrm{DP}=7,1$ anos; $62,5 \%$ feminino), com os mais diversos problemas de saúde (ex., doenças cardio-respiratórias, intervenções operatórias de baixa e média gravidades).

TABELA 1

Grupos amostrais de idosos do presente estudo

\begin{tabular}{|c|c|c|c|c|c|c|}
\hline & \multirow{2}{*}{ Coleta } & \multirow{2}{*}{$n$} & \multicolumn{2}{|c|}{ Homens } & \multicolumn{2}{|c|}{ Mulheres } \\
\hline & & & $N$ & $\%$ & $N$ & $\%$ \\
\hline Praticantes de atividade física & Coletiva & 62 & 12 & 19 & 50 & 81 \\
\hline Hospitalizados & Individual & 40 & 15 & 37 & 25 & 62 \\
\hline Usuários de centro de convivência & Coletiva & 97 & 29 & 30 & 68 & 70 \\
\hline Residentes em instituição de longa permanência & Individual & 64 & 58 & 91 & 6 & 9 \\
\hline Em tratamento para depressão & Individual & 48 & 20 & 42 & 28 & 58 \\
\hline
\end{tabular}


O terceiro grupo foi composto por 97 idosos que frequentavam um Centro de Convivência, também na cidade de Curitiba $(\mathrm{M}=68,7 ; \mathrm{DP}=6,7$ anos; 70,1\% feminino). Na sequência, o quarto grupo foi composto por 64 residentes de instituição de longa permanência (ILP) também da cidade de Curitiba ( $\mathrm{M}=70,4 ; \mathrm{DP}=7,8$ anos; $9,4 \%$ feminino). Por último, o grupo clínico foi composto por 48 pacientes de uma cidade do interior de Minas Gerais, avaliados em clínicas psiquiátricas e diagnosticados como diagnóstico primário de Episódio Depressivo Maior - EPD ( $\mathrm{M}=72,3$; $\mathrm{DP}=7,1$ anos; 58,3 $\%$ feminino), todos em tratamento medicamentosos, sendo que $46 \%$ também possuíam outras comorbidades (transtornos ansiosos, alcoolismo, dentre outros).

\section{Instrumento}

A EBADEP-ID possui 70 itens que rastreiam os sintomas da depressão, tais como descritos no DSM-5 e na CID-10, e pelas teorias cognitiva e comportamental da depressão. As respostas aos itens ocorrem no formato dicotômico ( $\operatorname{sim}$ ou não). No presente estudo, foi utilizada a versão reduzida de 32 itens de Carneiro, Baptista e Santos (2013), sendo retirados dois itens que apresentaram cargas fatoriais abaixo de 0,30. A consistência interna da escala foi bastante expressiva, sendo 0,95 pelo coeficiente alfa.

Apesar de se tratar de um instrumento reduzido, o conjunto de itens se manteve representativo dos diferentes domínios teóricos da depressão. Especificamente, a composição do instrumento foi de: 20 itens de descritor de tipo Cognitivo, quatro de humor, três vegetativos/somáticos, dois de natureza social e um motor. Vale ressaltar que o instrumento manteve 18 itens semanticamente positivos e 12 negativos, maximizando a cobertura do contínuo latente.

\section{Procedimento}

As coletas de dados ocorreram apenas após o projeto ter sido aprovado pelas instituições de coleta e pelo Comitê de Ética em Pesquisa da Universidade São Francisco (0422.0.142.000-11). Todos os participantes assinaram o Termo de Consentimento Livre e Esclarecido. Nas coletas que aconteceram de forma individual, o entrevistador leu as perguntas, e marcou as respostas conforme as informações dadas pelo sujeito.
Nas coletas em formato coletivo, os próprios idosos responderam ao questionário. A coleta teve duração média de 30 minutos.

\section{Análise de dados}

Os dados foram analisados utilizando o programa Mplus 7.1 (Muthén \& Muthén, 2017). Dada a natureza dicotômica dos indicadores, foi utilizado um estimador robusto para as análises fatoriais, o Weighted Least Squares Mean and Variance Adjusted (WLSMV). Para a produção de uma solução bifator, foi empregada a rotação bi-geomin, que produz um fator geral e $n$ fatores específicos, com a liberdade de existirem correlações entre esses fatores. $\mathrm{O}$ ajuste dos dois modelos testados - unidimensional e bifator - foi comparado com base no teste qui-quadrado e nos índices de ajuste Root Mean Square Error of Approximation (RMSEA; desejável abaixo de 0,08), Confirmatory Fit Index (CFI; desejável acima de 0,95) e Tuker-Lewis Index (TLI; desejável acima de 0,95). Uma análise suplementar da capacidade discriminativa do instrumento foi conduzida uma ANOVA para comparar os escores observados dos grupos.

\section{Resultados}

A etapa inicial das análises foi comparar o ajuste dos modelos unidimensional e bifator. Os resultados são apresentados na Tabela 2. Como se pode observar, apesar da significância estatística do teste qui-quadrado, ambos os modelos apresentaram um excelente ajuste aproximado aos dados. Ainda assim, o modelo bifator se mostrou aquele com melhor ajuste, $\Delta \chi^{2}(57)=198,93$, $p<0,05$.

As cargas fatoriais encontradas para ambas as soluções fatoriais foram analisadas e estão apresentadas na Tabela 3. O motivo de manter ambas as soluções foi comparar as cargas dos itens no fator geral de cada solução. Reise, Morizot e Hays (2007) sugerem que uma equivalência entre esses parâmetros nos dois modelos indica que os fatores específicos produzidos pela solução bifator são de menor importância. Ou seja, eles não capturam uma parcela substancial de variância comum, sendo, portanto, de pouca vantagem teórica em relação a uma solução simplesmente unidimensional.

TABELA 2

Índices de ajuste dos modelos fatoriais (EBADEP-ID 30 itens)

\begin{tabular}{lcccccc}
\hline & $\chi^{2}$ & $g l$ & $p$ & $R M S E A$ & $C F I$ & $T L I$ \\
\hline Unidimensional & 595,151 & 405 & $<0,001$ & 0,039 & 0,982 & 0,983 \\
Bifator & 396,221 & 348 & 0,0380 & 0,021 & 0,996 & 0,995 \\
\hline
\end{tabular}


TABELA 3

EBADEP-ID versão 30 itens e cargas fatoriais

\begin{tabular}{|c|c|c|c|c|}
\hline \multirow{2}{*}{ Itens } & \multirow{2}{*}{1 fator } & \multicolumn{3}{|c|}{ Bifator } \\
\hline & & Geral & $F 1$ & $F 2$ \\
\hline Sinto-me cada vez mais sozinho(a) & 0,74 & 0,73 & 0,10 & 0,27 \\
\hline Sinto-me menos capaz para realizar tarefas & 0,60 & 0,61 & 0,10 & $-0,11$ \\
\hline Tenho sentido vontade de chorar & 0,71 & 0,71 & 0,29 & $-0,13$ \\
\hline Faço planos para o futuro & $-0,65$ & $-0,64$ & 0,37 & $-0,07$ \\
\hline Estou me sentindo mais triste & 0,90 & 0,90 & 0,26 & 0,04 \\
\hline Estou acreditando menos em mim & 0,80 & 0,81 & $-0,02$ & $-0,01$ \\
\hline Sinto-me feliz com minha vida & $-0,85$ & $-0,84$ & 0,22 & 0,00 \\
\hline Acredito que atualmente nada vai bem na minha vida & 0,68 & 0,66 & $-0,03$ & 0,36 \\
\hline Comecei a inventar desculpas para ficar em casa sozinho(a) & 0,80 & 0,80 & $-0,10$ & $-0,05$ \\
\hline Compareço a festas e reuniões quando sou convidado(a) & $-0,72$ & $-0,70$ & 0,20 & $-0,30$ \\
\hline Consigo me concentrar nas atividades & $-0,84$ & $-0,84$ & 0,24 & 0,02 \\
\hline Estou me sentindo mais agitado(a) do que antes & 0,67 & 0,67 & 0,39 & 0,01 \\
\hline Prefiro a solidão & 0,72 & 0,71 & $-0,25$ & 0,23 \\
\hline Tenho me sentido angustiado(a) & 0,84 & 0,84 & 0,26 & $-0,11$ \\
\hline Minha vida está cada vez pior & 0,82 & 0,79 & $-0,01$ & 0,49 \\
\hline Morrer é a solução para os problemas & 0,62 & 0,59 & 0,03 & 0,48 \\
\hline Gosto da minha vida & $-0,94$ & $-0,93$ & 0,21 & 0,02 \\
\hline Gosto de mim & $-0,95$ & $-0,94$ & 0,20 & 0,09 \\
\hline Pensar no futuro me desanima & 0,88 & 0,88 & 0,09 & 0,08 \\
\hline Sinto-me com disposição & $-0,92$ & $-0,93$ & $-0,01$ & 0,24 \\
\hline Venho comendo normalmente & $-0,77$ & $-0,78$ & 0,10 & 0,10 \\
\hline Meus dias têm sido vazios & 0,88 & 0,88 & 0,06 & 0,18 \\
\hline Tenho pensado em me machucar & 0,71 & 0,72 & 0,19 & $-0,00$ \\
\hline Venho me sentido culpado(a) pelos problemas & 0,84 & 0,85 & 0,10 & 0,00 \\
\hline Consigo dormir à noite inteira & $-0,66$ & $-0,67$ & $-0,14$ & 0,12 \\
\hline Meus dias tem sido bons & $-0,92$ & $-0,91$ & 0,20 & $-0,06$ \\
\hline Viver está cada vez mais difícil & 0,75 & 0,74 & 0,18 & 0,23 \\
\hline Sinto prazer em realizar minhas atividades & $-0,91$ & $-0,90$ & 0,13 & 0,19 \\
\hline Sinto-me menos útil & 0,81 & 0,80 & 0,15 & 0,14 \\
\hline Continuo fazendo bem as minhas tarefas/atividades & $-0,75$ & $-0,74$ & 0,39 & 0,15 \\
\hline
\end{tabular}

Todos os itens apresentaram cargas expressivas no fator geral $(\geq 0,59)$, em ambos os modelos testados. Além disso, houve uma alta congruência entre as cargas nos fatores gerais das soluções unidimensional e bifator, sendo a correlação entre essas cargas $r=1,00$, $p<0,001$. Em outras palavras, apesar de o ajuste da solução bifator ter sido melhor aos dados, esse modelo foi praticamente equivalente ao modelo unidimensional, que representa uma explicação mais simples dos dados. Outra maneira de avaliar o mérito de manter fatores específicos é a partir das cargas dos itens nesses fatores. Dos 30 itens analisados, apenas seis deles carregaram $>0,30$ em um dos dois fatores específicos do modelo bifator. Isso evidencia que o conjunto de indicadores é mais informativo quanto a esse fator geral, sustentando a unidimensionalidade da EBADEP-ID.
Na sequência, foi investigada a fidedignidade dos 30 itens da EBADEP-ID. A consistência interna para os 30 itens ao assumir a unidimensionalidade dos itens foi alta, sendo 0,95 pelo coeficiente alfa $(0,98$ ao derivar o alfa de correlações tetracóricas entre os itens) e 0,98 pelo coeficiente ômega. Uma análise mais pormenorizada da fidedignidade foi conduzida por meio da inspeção da curva de informação total do teste, apresentada na Figura 1. Conforme o esperado para um teste que avalia sintomas de natureza psicopatológica, a curva apresentou um pico de informação localizado na porção superior do traço latente, próximo a um desvio-padrão acima da média. Isso indica que, de fato, o instrumento proporciona uma avaliação mais precisa para os indivíduos que apresentam níveis patológicos do construto. 


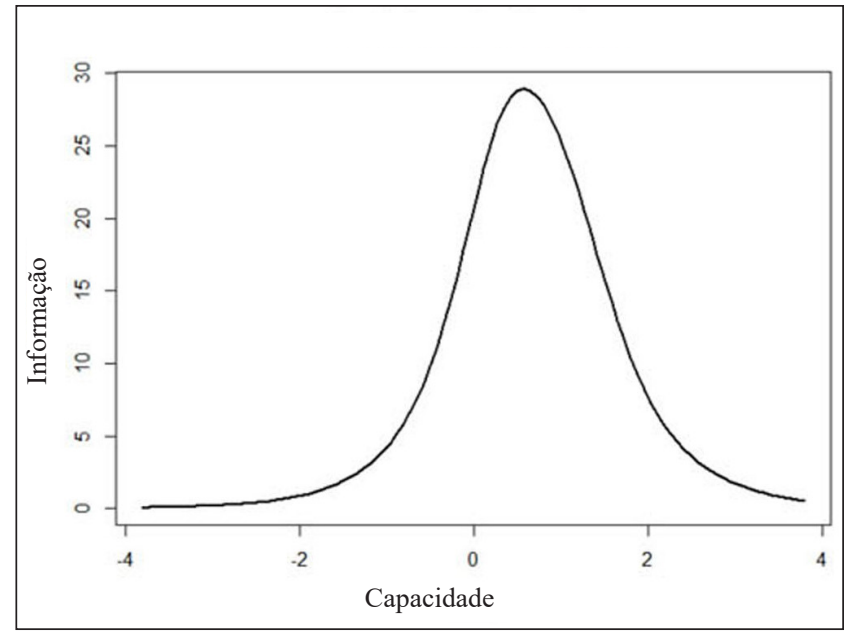

Figura 1. Curva de informação do teste.

Uma análise complementar de validade do instrumento foi feita a partir da comparação dos escores dos diferentes grupos amostrais na EBADEPID e da análise de sensibilidade e especificidade do teste. A ANOVA apontou diferenças significativas entre os grupos $F(4,305)=214,80, p<0,001)$. A média mais alta ocorreu para o grupo de indivíduos em tratamento para depressão 25,89 , seguido pelos indivíduos hospitalizados $(11,30)$, asilares $(7,73)$, praticantes de atividade física $(4,76)$ e usuários de centro de convivência $(4,44)$. O teste post hoc de Tuckey apontou diferença significativas entre quatro dos cinco grupos, apenas não havendo diferenças entre os dois últimos grupos. A análise de especificidade, por sua vez, foi feita comparando-se os grupos extremos de idosos saudáveis (praticantes de atividades físicas e usuários de centros de convivência) e idosos em tratamento para depressão. A diferença entre os dois grupos foi extrema $[t(204)=31,54, p<0,001, d=5,73]$, sendo a área debaixo da curva igual a $1,00, p<0,001$. $\mathrm{O}$ ponto de corte de 12 pontos ou mais resultou em uma sensibilidade de 1,00 e especificidade de 0,91.

\section{Discussão}

O presente estudo teve como objetivo realizar uma análise bifator do instrumento EBADEP-ID, uma escala de autorrelato de sintomas de depressão destinada à população de idosos. Para tanto, este modelo foi comparado, em termos de ajuste aos dados, a um modelo simples unidimensional. Embora o modelo bifator tenha apresentado melhor ajuste, verificou-se que poucos itens de fato carregaram nos fatores específicos, sendo as cargas no fator geral muito semelhantes às cargas da solução unidimensional. Conforme as indicações de Reise et al. (2007), esse resultado sustenta a unidimensionalidade da EBADEPID. Embora o instrumento seja composto por itens que endereçam uma série de aspectos clínicos específicos, o conjunto dos itens é mais discriminativo para um fator geral de depressão. Assim, respondendo ao questionamento que inspirou a presente investigação, o formato mais recomendado de cômputo de escores para a EBADEP-ID é o de um simples escore geral de sintomas de depressão. Esse achado é coerente com o que foi encontrado para as demais versões da EBADEP (Baptista, 2012; Batista, no prelo), e também com outros instrumentos desenvolvidos fora do Brasil, tal como o BDI-II (Brouwer et al., 2013).

Os resultados da análise fatorial possuem dois significados dignos de nota. Em primeiro lugar, o fator geral de depressão encontrado representa uma causa latente comum aos diversos sinais e sintomas da depressão. Essa perspectiva de modelagem e de entendimento teórico da depressão é conhecida como "modelo reflexivo", ou seja, aquele em que a variância dos indicadores ocorre enquanto um reflexo ou efeito da variável latente (Edwards \& Bagozzi, 2000). O fator de depressão aqui descrito representa uma variável que atua, nos indivíduos, como uma propensão geral a desenvolver os mais variados sinais e sintomas de depressão, sintetizando o conjunto de processos cognitivos e afetivos subjacentes ao transtorno. Em segundo lugar, os achados são consistentes com uma visão dimensional da depressão. Embora não seja consenso que a depressão é um fenômeno dimensional em vez de categórico (Cf. Pepper \& Nieuwsma, 2006), fenômenos psicopatológicos frequentemente se manifestam enquanto níveis (Haslam, Holland, \& Kuppens, 2012). A abordagem dimensional da depressão permite distinguir os indivíduos em termos de níveis de funcionamento depressivo, em vez de apenas separar entre "depressivos" e "não depressivos". Dessa forma, a EBADEP-ID permite captar variados graus de depressão, incluindo níveis subclínicos, considerados dignos de atenção em serviços de saúde (Solomon, Haaga, \& Arnow, 2001).

O escore geral proporcionado pela EBADEP-ID apresentou elevada fidedignidade, a partir de duas perspectivas de análise. Na primeira delas, os 30 itens obtiveram elevada consistência interna com base em três diferentes estimativas de consistência interna (alfa, alfa policórico e ômega). Na segunda delas, os itens se mostraram adequados ao uso em grupos clínicos, a partir da curva de informação. Além de a curva de informação ter se mostrado abrangente de um amplo espectro de escores acima e abaixo da média 
populacional, o pico da curva ocorreu em uma faixa característica de um funcionamento patológico (por volta de um desvio-padrão acima da média). Isso significa que o máximo de precisão ocorre justamente para indivíduos com maior sofrimento psicológico, como aqueles típicos de contextos clínicos (American Psychiatric Association, 2013).

Os escores gerais do instrumento foram utilizados para comparar os diferentes grupos amostrais incluídos no presente estudo. A EBADEP-ID se mostrou capaz de diferenciar todos os grupos de indivíduos com características distintas. Como seria esperado, idosos em tratamento para depressão foram aqueles que obtiveram as maiores médias, enquanto idosos considerados saudáveis (praticantes de atividades físicas e usuários de centros de convivência), foram aqueles com as menores médias. As diferenças entre esses dois grupos extremos foram de mais de cinco desvios-padrão ( $d$ de Cohen), o que caracteriza uma separação de grande magnitude. Vale ressaltar que outros dois grupos intermediários também se diferenciaram (hospitalizados e asilares), o que demonstra a boa capacidade discriminativa do instrumento para níveis intermediários de sintomas de depressão.

$\mathrm{O}$ uso de um ponto de corte no instrumento não é, necessariamente, antagônico a uma perspectiva dimensional da depressão. No presente caso, a proposta de um ponto de corte para a EBADEP-ID consiste apenas em fornecer um indicador útil para a decisão sobre a necessidade de uma avaliação mais apurada do paciente. É digno de nota que abordagens mais recentes de modelagem de aspectos da saúde mental têm integrado a ideia de contínuo e categoria, de modo que não são visões excludentes sobre um mesmo fenômeno psicológico (Masyn, Henderson, \& Greenbaum, 2010). Isso significa que pode existir um fator geral de depressão ao mesmo tempo em que existem dois ou mais grupos de indivíduos com um funcionamento qualitativamente distinto, que se diferenciam em termos de escores médios nesse fator (e.g., sem depressão, depressão leve, depressão moderada e severa). Essa hipótese pode ser testada diretamente com um modelo fatorial de misturas de populações, algo que fica como uma sugestão para estudos futuros com a EBADEPID - e também com outros instrumentos de depressão.

Uma análise secundária realizada, mas de grande utilidade clínica, é a capacidade diagnóstica da EBADEP-ID. Quando considerados os grupos de idosos saudáveis e idosos em tratamento para depressão, a escala alcançou índices de sensibilidade e especificidade bastante elevados (1,00 e 0,91, respectivamente). Esse achado em particular sustenta o uso da EBADEP-ID como uma robusta ferramenta de rastreio de sintomas de depressão. Um ponto de corte de 12 pontos é sugerido para fins de rastreio e classificação em contextos de avaliação em saúde mental.

Duas limitações do presente estudo devem ser mencionadas. A primeira é que as comparações entre os diferentes grupos foram feitas sem um teste da invariância dos parâmetros. Estudos subsequentes deverão, portanto, investigar a possibilidade de funcionamento diferencial dos itens da EBADEP-ID em diferentes grupos populacionais. A segunda é que o presente estudo prescindiu de uma medida externa padronizada de depressão. Mais especificamente, na composição do grupo clínico (idosos em tratamento), foram utilizados diferentes métodos clínicos para estabelecer a hipótese diagnóstica de depressão, sendo as avaliações feitas por variados profissionais clínicos. Seria importante não apenas avaliar o nível de correlação da EBADEP-ID com outras ferramentas padronizadas de avaliação da depressão (e.g., critérios do DSM-5), mas também verificar se as diferenças (d) observadas entre os grupos são similares entre as medidas. Isso serviria como uma evidência adicional de que o fator geral avaliado pela EBADEP-ID de fato representa os principais processos latentes da depressão tal como descritos nos manuais nosográficos (American Psychiatric Association, 2013).

\section{Considerações finais}

O presente estudo descreve achados que sustentam a EBADEP-ID como uma ferramenta útil no rastreio e detecção da depressão em idosos brasileiros. $\mathrm{O}$ instrumento apresentou-se unidimensional e com ótimas propriedades psicométricas, tais como elevada fidedignidade, sensibilidade e especificidade. Considerando o gradativo envelhecimento da população brasileira, um instrumento como a EBADEP-ID cumpre o importante papel de auxiliar profissionais da saúde envolvidos na avaliação e identificação de sofrimento psicológico de indivíduos longevos. 


\section{Referências}

Achterberg, W., Pot, A. M., Kerkstra, A., \& Ribbe, M. (2006). Depressive symptoms in newly admitted nursing home residents. International Journal of Geriatric Psychiatry, 21(12), 1156-1162. https://doi.org/10.1002/gps.1623

Almeida, O. P., Forlenza, O. V, Lima, N. K., Bigliani, V., Arcuri, S. M., Gentile, M., ... de Oliveira, D. A. (1997). Psychiatric morbidity among the elderly in a primary care setting-report from a survey in São Paulo, Brazil. International Journal of Geriatric Psychiatry, 12(7), 728-36. Retrieved from http://www.ncbi.nlm.nih.gov/ pubmed/9251935. https://doi.org/10.1002/(sici)1099-1166(199707)12:7<728::aid-gps624>3.0.co;2-c

American Psychiatric Association. (2013). Diagnostic and Statistical Manual of Mental Disorders (5 ${ }^{\text {th }}$ ed.). Washington, DC: American Psychiatric Publishing.

Argimon, I. I. de L., Paloski, L. H., Farina, M., \& Irigaray, T. Q. (2016). Aplicabilidade do Inventário de Depressão de Beck-II em idosos: uma revisão sistemática. Avaliação Psicológica, 15(Edição Especial), 11-17. https://doi. org/10.15689/ap.2018.1703.14651.07

Baptista, M. N. (2012). Escala Baptista de Depressão Versão Adulto (EBADEP-A). São Paulo, SP: Vetor. https://doi. org/10.1590/s1413-82712012000300007

Baptista, M. N. (no prelo). Escala Baptista de Depressão (versão Infanto-Juvenil) EBADEP-IJ. (DOI INEXISTENTE)

Baptista, M. N. \& Borges, L. (2016). Revisão integrativa de instrumentos de depressão em crianças/adolescentes e adultos na população brasileira. Avaliação Psicológica, 15(Edição Especial), 19-32. https://doi.org/10.15689/ ap.2016.15ee.03

Baptista, M. N., Hauck Filho, N., \& Cardoso, C. (2016). Depressão e bem-estar subjetivo em crianças e adolescentes: teste de modelos teóricos. Psico, 47(4), 259-267. https://doi.org/10.15448/1980-8623.2016.4.23012

Beck, A. T., Rush, A. J., Shaw, B. F., \& Emery, G. (1987). Terapia Cognitiva da Depressão. Porto Alegre, RS: Artmed. (DOI INEXISTENTE)

Beck, A. T., Ward, C. H., Mendelson, M., Mock, J., \& Erbaugh, J. (1961). An inventory for measuring depression. Archives of General Psychiatry, 4, 561-71. Retrieved from http://www.ncbi.nlm.nih.gov/pubmed/13688369. (DOI INEXISTENTE)

Borges, L., Baptista, M. N., \& Serpa, A. L. O. (2017). Structural Analysis of Depression Indicators Scale-Children and Adolescents (BAID-IJ): A Bifactor-ESEM Approach. Temas Em Psicologia, 25(2), 545-552. https://doi. org/10.9788/tp2017.2-08

Bottino, C. M. C., Blay, S. L., \& Laks, J. (2012). Diagnóstico e Tratamento dos Transtornos do Humor em Idosos. São Paulo, SP: Atheneu. (DOI INEXISTENTE)

Brouwer, D., Meijer, R. R., \& Zevalkink, J. (2013). On the factor structure of the Beck Depression Inventory-II: $\mathrm{G}$ is the key. Psychological Assessment, 25(1), 136-145. https://doi.org/10.1037/a0029228

Burns, A., Höfer, S., Curry, P., Sexton, E., \& Doyle, F. (2014). Revisiting the dimensionality of the Hospital Anxiety and Depression Scale in an international sample of patients with ischaemic heart disease. Journal of Psychosomatic Research, 77(2), 116-121. https://doi.org/10.1016/j.jpsychores.2014.05.005

Carneiro, A. M., Baptista, M. N., \& Santos, A. A. A. (2013). Medidas de sintomas depressivos em idosos: relações com variáveis sociodemográficas. Avances en Psicología Latinoamericana, 31(3), 483-492. Retrieved from http:// www.scielo.org.co/scielo.php?script=sci_arttext\&pid=S1794-47242013000300003\&lng=en\&nrm=iso\&tlng=pt. https://doi.org/10.26823/revistadonufen.vo19.n02translation12

Chin, W. Y., Choi, E. P. H., Chan, K. T. Y., Wong, C. K. H., Kwong, D., \& Tsang, J. (2015). The Psychometric Properties of the Center for Epidemiologic Studies Depression Scale in Chinese Primary Care Patients: Factor Structure, Construct Validity, Reliability, Sensitivity and Responsiveness. PLOS ONE, 10(8). https://doi. org/10.1371/journal.pone.0135131

Coutinho, F. L., Hamdan, A. C., \& Baptista, M. N. (2016). Escala Baptista de Depressão para Idosos - EBADEP-ID: evidências de validade. Perspectivas En Psicología, 13(2), 1-9. https://doi.org/10.1590/s1413-82712011000200004

da Silva, J. V. \& Baptista, M. N. (2016). Vitor Quality of Life Scale for the Elderly: evidence of validity and reliability. SpringerPlus, 5(1), 1450-1462. https://doi.org/10.1186/s40064-016-3130-4

Edwards, J. R., \& Bagozzi, R. P. (2000). On the nature and direction of relationships between constructs and measures. Psychological Methods, 5(2), 155-174. 
Ferster, C. B., Culbertson, S., \& Boren, C. P. (1997). Princípios do comportamento. São Paulo, SP: Hucitec. (DOI INEXISTENTE)

Gomez, R. \& McLaren, S. (2015). The Center for Epidemiologic Studies Depression Scale: Support for a Bifactor Model With a Dominant General Factor and a Specific Factor for Positive Affect. Assessment, 22(3), 351-360. https://doi.org/10.1177/1073191114545357

Hamilton, M. (1967). Development of a rating scale for primary depressive illness. The British Journal of Social and Clinical Psychology, 6(4), 278-96. Retrieved from http://www.ncbi.nlm.nih.gov/pubmed/6080235. https://doi.org/10.1111/j.2044-8260.1967.tb00530.x

Haslam, N., Holland, E., \& Kuppens, P. (2012). Categories versus dimensions in personality and psychopathology: a quantitative review of taxometric research. Psychological Medicine, 42(5), 903-20. https://doi.org/10.1017/ s0033291711001966

Iani, L., Lauriola, M., \& Costantini, M. (2014). A confirmatory bifactor analysis of the hospital anxiety and depression scale in an Italian community sample. Health and Quality of Life Outcomes, 12(1), 84-91. https://doi. org/10.1186/1477-7525-12-84

Instituto Brasileiro de Geografia e Estatística. (2002). Perfil dos Idosos Responsáveis pelos Domicílios. Retrieved from http://www.ibge.gov.br/home/presidencia/noticias/25072002pidoso.shtm. (DOI INEXISTENTE)

Kim, G., DeCoster, J., Huang, C.-H., \& Bryant, A. N. (2013). A meta-analysis of the factor structure of the Geriatric Depression Scale (GDS): the effects of language. International Psychogeriatrics, 25(1), 71-81. https://doi. org/10.1017/s1041610212001421

Masyn, K. E., Henderson, C. E., \& Greenbaum, P. E. (2010). Exploring the Latent Structures of Psychological Constructs in Social Development Using the Dimensional-Categorical Spectrum. Social Development, 19(3), 470-493. https://doi.org/10.1111/j.1467-9507.2009.00573.x

Montgomery, S. A. \& Åsberg, M. (1979). A new depression scale designed to be sensitive to change. The British Journal of Psychiatry, 134(4). Retrieved from http://bjp.rcpsych.org/content/134/4/382. https://doi.org/10.1192/ bjp.134.4.382

Muthén, L. K. \& Muthén, B. O. (2017). Mplus user's guide (7ạ ed.). Los Angeles, CA: Muthén \& Muthén.

Norton, S., Cosco, T., Doyle, F., Done, J., \& Sacker, A. (2013). The Hospital Anxiety and Depression Scale: A meta confirmatory factor analysis. Journal of Psychosomatic Research, 74(1), 74-81. https://doi.org/10.1016/j. jpsychores.2012.10.010

Organização Mundial de Saúde. (1995). Classificação dos Transtornos Mentais e de Comportamento da CID-10. Porto Alegre, RS: Artmed. https://doi.org/10.1590/s1516-44461999000200014

Osman, A., Barrios, F. X., Gutierrez, P. M., Williams, J. E., \& Bailey, J. (2008). Psychometric properties of the Beck Depression Inventory-II in nonclinical adolescent samples. Journal of Clinical Psychology, 64(1), 83-102. https:// doi.org/10.1002/jclp.20433

Osman, A., Wong, J. L., Bagge, C. L., Freedenthal, S., Gutierrez, P. M., \& Lozano, G. (2012). The Depression Anxiety Stress Scales-21 (DASS-21): Further Examination of Dimensions, Scale Reliability, and Correlates. Journal of Clinical Psychology, 68(12), 1322-1338. https://doi.org/10.1002/jclp.21908

Pepper, C. M. \& Nieuwsma, J. A. (2006). Issues in the measurement of depression: Purpose, population, and interpretation. Measurement: Interdisciplinary Research and Perspectives, 4(3), 165-169. (DOI INEXISTENTE)

Pocklington, C., Gilbody, S., Manea, L., \& McMillan, D. (2016). The diagnostic accuracy of brief versions of the Geriatric Depression Scale: a systematic review and meta-analysis. International Journal of Geriatric Psychiatry, 31(8), 837-857. https://doi.org/10.1002/gps.4407

Polyakova, M., Sonnabend, N., Sander, C., Mergl, R., Schroeter, M. L., Schroeder, J., \& Schönknecht, P. (2014). Prevalence of minor depression in elderly persons with and without mild cognitive impairment: A systematic review. Journal of Affective Disorders, 152-154, 28-38. https://doi.org/10.1016/j.jad.2013.09.016

Reise, S. P., Moore, T. M., \& Haviland, M. G. (2010). Bifactor Models and Rotations: Exploring the Extent to Which Multidimensional Data Yield Univocal Scale Scores. Journal of Personality Assessment, 92(6), 544-559. https://doi. org/10.1080/00223891.2010.496477

Reise, S. P., Morizot, J., \& Hays, R. D. (2007). The role of the bifactor model in resolving dimensionality issues in health outcomes measures. Quality of Life Research, 16(Suppl 1), 19-31. https://doi.org/10.1007/s11136-0079183-7 
Santor, D. A., Gregus, M., \& Welch, A. (2006). Eight Decades of Measurement in Depression. Measurement: Interdisciplinary Research \& Perspective, 4(3), 135-155. https://doi.org/10.1207/s15366359mea0403_1

Schmittmann, V. D., Cramer, A. O. J., Waldorp, L. J., Epskamp, S., Kievit, R. A., \& Borsboom, D. (2013). Deconstructing the construct: A network perspective on psychological phenomena. New Ideas in Psychology, 31(1), 43-53. https://doi.org/10.1016/j.newideapsych.2011.02.007

Shafer, A. B. (2006). Meta-analysis of the factor structures of four depression questionnaires: Beck, CES-D, Hamilton, and Zung. Journal of Clinical Psychology, 62(1), 123-146. https://doi.org/10.1002/jclp.20213

Silberman, C., Souza, C., Wilhems, F., Kipper, L., Wu, V., Diogo, C., ..., \& Chaves, M. (1995). Cognitive deficit and depressive symptoms in a community group of elderly people: a preliminary study. Revista de Saúde Pública, 29(6), 444-450. https://doi.org/10.1590/s0034-89101995000600005

Solomon, A., Haaga, D. A., \& Arnow, B. A. (2001). Is clinical depression distinct from subthreshold depressive symptoms? A review of the continuity issue in depression research. The Journal of Nervous and Mental Disease, 189(8), 498-506. Retrieved from http://www.ncbi.nlm.nih.gov/pubmed/11531201. https://doi. org/10.1097/00005053-200108000-00002

Spearman, C. (1904). “General intelligence,” objectively determined and measured. The American Journal of Psychology, 15(2), 201-292. https://doi.org/10.2307/1412107

Stanley, I. H., Hom, M. A., Rogers, M. L., Hagan, C. R., \& Joiner, T. E. (2016). Understanding suicide among older adults: a review of psychological and sociological theories of suicide. Aging \& Mental Health, 20(2), 113-122. https://doi.org/10.1080/13607863.2015.1012045

Volkert, J., Schulz, H., Härter, M., Wlodarczyk, O., \& Andreas, S. (2013). The prevalence of mental disorders in older people in Western countries - a meta-analysis. Ageing Research Reviews, 12(1), 339-353. https://doi.org/10.1016/j. arr.2012.09.004

Walker, J., Burke, K., Wanat, M., Fisher, R., Fielding, J., Puntis, S., ..., \& Sharpe, M. (2016). How common is depression in general hospital inpatients? Findings from a worldwide systematic review. Journal of Psychosomatic Research, 85, 87-92. https://doi.org/10.1016/j.jpsychores.2016.03.217

World Health Organization. (2002). Mental Health in our World: The Challenges Ahead. Retrieved from http://www. who.int/director-general/speeches/2001/english/20011211_mentalhealthseminar.html. (DOI INEXISTENTE)

World Health Organization. (2013). Suicide Prevention. Retrieved from http://www.who.int/mental_health/ prevention/en/. (DOI INEXISTENTE)

Dados dos autores:

Makilim Nunes Baptista - Doutor, Universidade São Francisco.

Luísa Milani Santos - Graduanda, Universidade Estadual de Campinas.

Nelson Hauck Filho - Doutor, Universidade São Francisco.

\section{Endereço para correspondência:}

Makilim Nunes Baptista

Estrada da Rhodia, 7250 casa 33, Cond. Lagoa Serrena - Vila Holandia

13085-902, Campinas, São Paulo, Brasil

E-mail: makilim01@gmail.com

Recebido em: 19/02/2018.

Aceito em: 09/11/2018.

Publicado em: 05/08/2019. 